\title{
Pervasive refusal syndrome revisited: a conative disorder
}

\author{
Jan N. M. Schieveld ${ }^{1,2} \cdot$ Karl Sallin $^{3}$ \\ Published online: 21 November 2020 \\ (c) Springer-Verlag GmbH Germany, part of Springer Nature 2020
}

Keywords Pervasive refusal syndrome $\cdot$ Apathy $\cdot$ Catatonia $\cdot$ Conative $\cdot$ DSM-5 $\cdot$ ICD-11 $\cdot$ MESH term

Pervasive Refusal Syndrome (PRS) is a rare but serious child psychiatric disorder; its prevalence is unknown. A PubMed search on February 23, 2020 with the search terms: "Pervasive AND Refusal AND Syndrome" resulted in only 34 hits during the period 1988-2019, with finally only 31 hits valid dating from the period 1991-2019. PRS is characterized by a pervasive refusal of activities such as social engaging, speaking, eating and drinking and selfcare, and it is often accompanied by an active-and often angry-resistance to help. These are considered the core criteria (Table 1). This frequently leads to an endangered state of the child, and to exhaustion of parents as well as professionals involved (1). The seriousness of PRS also is reflected by the severe suffering of both the child and its family, and often requires intensive multidisciplinary (inpatient) hospital treatment periods $>6$ months. It frequently results in splitting mechanisms and a dividing of the treatment team, and last but not least: it is hard to diagnose, or not recognized at all.

Neither DSM-5 nor ICD-11 recognize PRS rendering it important to bring the syndrome to the attention of all disciplines constituting the pediatric mental health community. And that is why this month's paper by John Otasowie et al. [2] entitled. Pervasive refusal syndrome: systematic review of case reports" is very welcome (2). Its strengths are its thorough critical screening and discussing of the existent literature by using the PRISMA guidelines, its clear systematic structure and its honest approach regarding all the aspects which we do not know or which we do not understand (yet).

Jan N. M. Schieveld

jan.schieveld@mumc.nl

1 Department of Psycxhiatry and NeuroPsychology, Maastricht University Medical Center, Maastricht, The Netherlands

2 Mutsaers Academy, Venlo, The Netherlands

3 Karolinska Institute, Stockholm, Sweden
However, we also want to make some additional observations regarding six aspects: (1) the essence of the core feature of PRS and its overarching diagnostic category; (2) the clinical diagnostic problem of atypicality; (3) comorbidity; (4) primary versus secondary disorders; (5) differential diagnoses, and; (6) treatment.

Ad (1) The essence of PRS. The first six categories of the neuropsychiatric mental status examination are: appearance, consciousness and cognition, thinking, perception, mood and affect, will and (motor) behavior. Disorders of this last group are called "conative" disorders-from the Latin noun "conatus" which means effort/impulse/tendency. The classical—but nearly forgotten by neuropsychiatry, but not by psychology—conative symptoms are psychomotor retardation, psychomotor overactivity, motor stereotypy, catatonia, posturing, negativism, and all other disturbances of action and/or (motor) behavior not otherwise specified (such as apathy in e.g., residual/disorganized schizophrenia). Accordingly, PRS should be regarded primarily as a conative disorder because of its most important core feature: an active, and often angry, resistance against any verbal or non-verbal and or motor action/-interaction on all essential domains of human life. Frequently this occurs without a mood disorder, however: if this also is present, either right from the start or if it occurs during the course of the PRS, then the diagnostic separation between PRS and severe depression can be very difficult or even impossible. If the distinction is not possible, we infer a blended comorbidity problem (see ad 3 ).

(2) In clinical medicine and also in psychiatry atypicality is a common diagnostic issue. After all, human efforts in the systematic classification of symptoms and signs into core features of a clinical diagnosis are frequently frustrated by Mother Nature's own input or lack of it. So, in spite of our clear attempts to delineate and formulate core and additional criteria, in daily clinical practice the "core criteria are frequently met only partially (Table 1 ). 
Table 1 Adapted diagnostic criteria for PRS (From Jaspers et al., see [1])

\author{
Diagnostic criteria for pervasive refusal syndrome \\ A. Partial or complete refusal in three or more of the following domains: (1) eating, (2) mobilization, (3) \\ speech, (4) attention to personal care \\ B. Active and angry resistance to acts of help and encouragement \\ C. Social withdrawal and school refusal \\ D. No organic condition accounts for the severity of the degree of symptoms \\ E. No other psychiatric disorder could better account for the symptoms \\ F. The endangered state of the patient requires hospitalization
}

Core criteria are: $\mathrm{A}, \mathrm{B}$ and $\mathrm{C}$

Additional criteria are: $\mathrm{D}, \mathrm{E}$ and $\mathrm{F}$

From Reference 1 and by permission of the copyright owner
The common observation of atypicality plays a major role in the difficulties of diagnosing PRS.

(3) In addition, and especially in psychiatry, the issue of comorbidity warrants attention. A clinical disorder is frequently accompanied by another disorder or symptoms thereof, such as psychotic features/mood and or anxiety features/catatonic features/etc. This again complicates making the right diagnosis/diagnoses and leads to confusion and misunderstandings in scientific and clinical reasoning and decision making.

(4) Primary versus secondary disorders. This issue also plays a major role in neuropsychiatry e.g., in epilepsy or in delirium. Primary refers to an unknown cause, and secondary to a known underlying cause. Especially in psychiatry we know, and frequently encounter, this important clinical difference between a primary or secondary disorder such as in psychosis, major depressive disorder or the differential diagnosis of a conversion disorder. The secondary causes often appear to be in reaction to a trauma or major somatic cause or represent an attempt to avoid or gain something, first by developing and later by maintaining clinical symptoms. A classic example of the last is e.g., selective mutism in school. This often leads to extra attention of the parents at home and of the teacher at school and to a lack of verbal challenges in the classroom regarding study results or oral presentations. First this disorders starts insidiously, as a primary disorder, but once the child has experienced or discovered, often involuntarily and unconsciously, that this is an unexpected but successful strategy it will maintain its symptoms. And so, an insidious primary disorder develops into a secondary disorder. This issue probably also plays an important role in PRS, thus bringing to mind the old adagium, this old guiding principle, in child psychiatry: "Identify the stressors". In addition, we need to identify the maintaining factors (e.g., negative enforcement). At the same time, it is important to keep in mind that malingering or Munchausen-by-proxy may result in a behavior phenomenologically indistinguishable from core features of PRS.
(5) Differential diagnostic considerations are at the heart of good clinical reasoning as they help to protect us from a tunnel vision. This is of importance as again Mother Nature, and so also the phenomenology of neuropsychiatry, excels in mimicking. But how to find one's proper way in this mixture or maze of core symptoms, atypicality and comorbidities? Given the overall unfamiliarity with conative disorders, and a dearth in scientific and treatment knowledge and a refractoriness to a commonsense treatment approach there exists in the case of PRS frequently no golden route, neither towards the correct diagnosis nor towards the right clinical approach. And so there also is the risk of excessive investigations leading to only more problems including medical complications, e.g., bacterial infections, post lumbar puncture headache and anaphylaxis. Also psychological complications occur. The insecurity and anxiety evoked as a result of e.g., a small brain lesion detected in an MRI scan, or genetic variations of undecided significance has a negative impact in general, but in particular it introduces the hazard of rumination on unclear findings-vexing patients/parents and/or doctors-with a shift in focus eventually delaying the necessary next step as a result. The loss of pace amounts to treatment being withheld, and-at an additional financial cost. Thus, diagnostic criteria-and the widespread knowledge of these-may be very helpful (see e.g., Tables 3, 4 of the article of Jaspers et al. [1]).

(6) For treatment, psychotherapy of any modality and form, drugs and ECT are all used for PRS, but most of the time with disappointing results. Importantly, prior to the initiation of such interventions, first the question has to be answered for which precise indication? Which symptom(s) out of the whole spectrum of PRS is targeted? And in which order? Our impression is that the most useful treatment components up to now are "Tenderly Loving Care" in combination with a common-sense approach, a rehabilitation and counseling program for the child AND the parents AND other family members, guided by the three classic principles: (1) find the stressors and maintaining factors, (2) start low and go slow" and (3) take your time. 
As a final thought, we want to share with you this consideration: there still is a lot we do not know, nor understand, about PRS, but which we nevertheless have to learn. This is true also of other conative disorders, among which we recognize in particular catatonia, presently in DSM-5 a specifier to other know or unknown psychiatric or somatic disorders, and apathy. Apathy is a common neuropsychiatric syndrome defined by a lack of spontaneous or responsive (eye) contact, verbal and non -verbal communication, emotion and action (voluntary and/or goal-directed behavior). Most of the time it is not the patient who is complaining but partner, family and/or nurses.

And so we hope that the leading committees of the DSM-5 and the ICD-11, which both also do not recognize the word conative, (and neither does the U.S. National Library of Medicine regarding the potential MESH term "conative") will examine the pros and cons of incorporation of these clinically important-but sometimes very difficult and highly confusing-disorders including PRS into classification systems.

\section{Reference}

1. Jaspers T, Hanssen GM, van der Valk JA, Hanekom JH, van Well GT, Schieveld JN (2009) Pervasive refusal syndrome as part of the refusal-withdrawal-regression spectrum: critical review of the literature illustrated by a case report. Eur Child Adolesc Psychiatry 18(11):645-651. https://doi.org/10.1007/s00787-009-0027-6 (Epub 2009 May 21, In Open Access)

2. Otasowie J, Paraiso A, Bates G (2020) Pervasive refusal syndrome: systematic review of case reports. Eur Child Adolesc Psychiatry. https://doi.org/10.1007/s00787-020-01536-1 